\title{
Scientific evidence for the relationship between periodontitis and cardiovascular disease
}

\author{
Alonso A. Rosado ${ }^{1,2,3}$, Hernández G. Marcos ${ }^{1,4}$, Pérez R. Gómez ${ }^{1,5}$ \\ ${ }^{1}$ University of Salamanca, Salamanca, Spain \\ ${ }^{2}$ Complutense University, Madrid, Spain \\ ${ }^{3}$ Private Practice of Periodontology, Salamanca, Spain \\ ${ }^{4}$ Private Practice of Estomatology, Leon, Spain \\ ${ }^{5}$ Nursing Universitary School, Salamanca, Spain \\ Email: angelalonso1955@gmail.com
}

Received 22 May 2013; revised 23 June 2013; accepted 5 July 2013

Copyright (C) 2013 Rosado A. Alonso et al. This is an open access article distributed under the Creative Commons Attribution License, which permits unrestricted use, distribution, and reproduction in any medium, provided the original work is properly cited.

\begin{abstract}
A lot of clinical studies have investigated the possible association between periodontitis and coronary heart disease (CHD). Some of them indicate the existence of an epidemiologic association between these two concepts, and support previous investigations that have found that chronic periodontal inflammation, persistent bacterial infection with the presence of major periodontal pathogens, deep periodontal pockets. The number of missing teeth and other periodontal markers, seem to be important risk factors for cardiovascular diseases. Nevertheless, it will be required to carry out better controlled and larger studies to identify if these biological mechanisms are responsible for these increased risks and to provide a convincing support of a casual association and to determine if periodontal treatments could prevent CHD. Since periodontal disease and cardiovascular disease are common, their association has a significant public health importance. They share common risk factors, such as increasing age, smoking, stress, socioeconomic status and body fat metabolism, and that is the reason why there could be significant bias.
\end{abstract}

Keywords: Periodontitis; Cardiovascular Disease; Chronic Heart Disease; Periodontal Pathogens; Acute Myocardial Infarction; Arteriosclerosis

\section{INTRODUCTION}

Periodontitis is an infectious disease that affects the supporting tissues of the teeth. Its aetiology is multifactorial and its prevalence is estimated between $30 \%$ and $40 \%$ of the adult population. The pathogenesis is the result of an interaction between a biofilm predominantly composed by anaerobic gram-negative bacteria and some microaerophilic species and the host immune system [1].

Recent studies have shown that periodontal infection is associated and may play a contributory role in the development of serious systemic diseases, such as cardiovascular diseases, premature births and low weight newborns [2]. Thus, in a study of oral health and cardiovascular diseases conducted in the US with a sample of 44, 119 subjects, it was shown a statistically significant relationship between periodontal disease and an increased risk of atherosclerosis, myocardial infarction and stroke [3].

This relationship between periodontitis and cardiovascular disease awakens interest, and as a result, it could be necessary to control periodontitis as a new member on the list of risk factors for cardiovascular disease.

\section{PATHOPHYSIOLOGICAL INTERRELATION}

Periodontitis can cause haemostatic disorders such as increased plasma fibrinogen, white blood cells count, CRP, and viscosity of the blood. Moreover it has been found a relationship between the Von Willebrand factor (factor VIII), LPS and IL-1, which induced the liberation of this factor from endothelial cells, producing platelet aggregation and inflammatory foci where a thrombus can be generated [4].

Mattila, et al., in 1995, established that oral health is an index for predicting coronary disease and previous history of heart attacks and diabetes, because patients who suffered from acute myocardial infarction developed also periodontitis and showed a poorer oral health in comparison to healthy patients. They also observed 
that individuals with severe dental infections have higher levels of Von Willebrand factor antigen, leukocytes and fibrinogen [5].

\subsection{Relationship between Periodontitis and Ischaemic Heart Disease}

The main mechanism of action is caused by the effect that bacteria and their endotoxins produce into inflammatory reactions, haemostatic processes and alterations in lipid metabolism [6].

In the periodontal pocket, there is a large number of gram-negative bacteria which come into contact with the underlying tissue and periodontal blood vessels. From this periodontal infection a subclinical chronic bacteraemia is produced form which there is a periodic release of cytokines such as CRP, 1-antitrypsin, haptoglobin, fibrinogen, thromboxanes, IL-1, 6, 8 and TNF, which also pass into the general circulation. All these factors can initiate platelet adhesion and aggregation, promoting the formation of foam cells and accumulation of cholesterol in the arterial intima, which promotes arteriosclerosis and thrombosis, so that a coronary disease would be likely to appear [7].

Another mechanism that has been considered is the immune response as reaction to periodontitis varies among individuals and may be due to genetic differences, including secretory capacity of monocytes. These monocytes play a critical role in the formation of atheroma, with the risk of thromboembolism, which may relate ischaemic heart disease to periodontitis [8].

\subsection{Which Bacteria Seem to be Involved?}

The damage generated by periodontitis in the epithelium contributes the passage of bacteria into the bloodstream, producing transitory bacteraemia. During bacteraemia more than 30 species of bacteria were found among which predominates Viridans streptococci, responsible for diseases such as rheumatic fever, valvular heart disease and bacterial endocarditis [9].

Streptococcus sanguis is a commensal bacteria of the oral flora, which belongs to the group of Viridans streptococci and when it gets into the bloodstream it becomes a potent thrombogenic agent as it is able to induce platelet adhesion and aggregation through a cross reaction, simulating the binding sites of collagen type I and II [10, 11].

Porphyromonas gingivalis is an anaerobic gramnegative periodontopathogenic, which has fimbriae on its surface, that allow it to adhere and invade epithelial and endothelial cells, multiplying within them, evading the immune response and altering their normal function [1013].

In a study about $P$. gingivalis published in Circulation in 2002, Li, et al., commented that epidemiological data suggest that dental infections such as periodontitis can spread through the circulatory system and thus participate in atherosclerotic progression. In this study, it was proposed to investigate the effects of repeated systemic inoculations with $P$. gingivalis in the progression of atherosclerosis in heterozygous mice having a deficit of apolipoprotein E (Apo E).

Mice were inoculated intravenously with $P$. gingivalis alive once a week for 10, 14 or 24 consecutive weeks. After 24 weeks, the aortic injury was greater in the inoculated ones than in non-inoculated mice. Ribosomal DNA of $P$. gingivalis was found in the aortas, liver and heart, 24 weeks after inoculation. These results provide evidence that $P$. gingivalis accelerates the progression of atherogenic plaque [14].

Similarly, it has been observed that periodontal pathogens as A. actinomycetemcomitans have been implicated in endocarditis processes, possibly because of its ability to adhere to damaged heart valves $[15,16]$.

\subsection{Is There a Relationship between Periodontal Disease and Cardiovascular Disease?}

Beck, et al. (1996) observed that a bigger than a $3 \mathrm{~mm}$ depth tube in a periodontal pocket, generalized throughout the mouth, increases the risk of ischemic heart disease. As they explained, there is a strong correlation between the level of periodontal bone loss and myocardial infarction, which increases the risk of thromboembolic phenomena [17].

Emingil, et al. (2000) found an association between periodontitis and myocardial infarction, with widespread presence of periodontal pockets larger than or equal to 4 $\mathrm{mm}$ in $45 \%$ of patients with a history of stroke, and $25 \%$ in controls [18].

De Stefano, et al. (1993) conducted a study that included 9760 individuals between 25 and 74 years old, whom have been controlled some factors associated with coronary heart disease; the results after a 14-year monitoring periodontal disease was associated with an increase of $25 \%$ in cardiovascular disease risk. This relationship was stronger in individuals under 50 suffering periodontitis. Also they stated that individuals with high bone loss show an increase of $50 \%$ in the incidence of ischemic heart disease [7].

Loesche, et al. (1998) established an association between the number of missing teeth and the coronary heart disease; thus $53 \%$ of edentulous patients had coronary heart disease [19].

Takata, et al. (2001) described a predictive relationship between the number of missing teeth and the risk of ischemic heart disease in octogenarians. Those with 20 or more teeth present in the mouth have less risk of 
ischemic heart disease. Nevertheless it was found no linear relationship between the number of missing teeth and abnormalities in electrocardiograms [20].

Hung, et al. (2003) examined the association between oral health and peripheral arterial disease (PAD). They conducted a prospective study of 45,136 men free of cardiovascular disease. During the 12 years of monitoring they identified 342 cases of PAD. It resulted that there is a significant association between tooth loss and $\mathrm{PAD}$, especially in men with periodontal disease [21].

Meurman, et al. (2004) consider that this chronic infection is the pathogenesis of cardiovascular disease through the release of cytokines and other inflammatory mediators such as C-reactive protein and tumour necrosis factor, which can initiate a cascade of biochemical reactions and cause epithelial damage, facilitating the accumulation of cholesterol plaque. However, due to the multifactorial nature of both diseases, is difficult to confirm a causal association and the published results are controversial [12].

Hung, et al. (2003) also evaluated the relationship of missing teeth with the incidence of coronary heart disease in two cohort studies that concluded a significant association [21].

Joshipura, et al. (2003) conducted a study to examine the incidence of coronary heart disease in relation to the number of present teeth and periodontal disease and to explore potential mediators of this association in a cohort study. They concluded that tooth loss may be associated with an increased risk of coronary heart disease among subjects with a positive history of periodontal disease, being the diet a mediator in the relationship [22].

Pussinen, et al. (2003) studied whether antibodies against periodontal pathogens may be associated with periodontal disease. Evidence suggests that chronic infections increase atherogenesis and the risk of coronary heart disease. Pathogens such as A. actinomycetemcomitans and P. gingivalis along with certain clones of $A$. actinomycetemcomitans were found in atherogenesis plaques so they could have a particularly potential role in the production of non-oral infections.

The immune system response to periodontitis may be normal or elevated against certain bacteria from periodontal disease.

The aim was to study in a random group of patients their levels of antibodies against periodontal pathogens and their association with coronary artery disease. The association of antibodies against periodontal pathology establishes a risk factor for coronary heart disease.

The study sample comprised 2000 people aged between 45 and 74 years, of which 1163 were men and of these 159 (14\%) had coronary artery disease.

A comprehensive questionnaire was made and personal and medical data were collected (number of teeth, fillings, lipid levels, fibrinogens, cholesterol).

Seropositive individuals were identified as those who had antibody levels above 0.5 EU (ELISA measurement units) against $A$. actinomycetemcomitans and $P$. gingivalis, and the values obtained were multiplied by 1.5 for each risk factor.

Survey results show that in the group of middle-aged men, coronary artery disease subjects had significantly fewer teeth than subjects without coronary disease (12.2 against 17.7). Among subjects toothed, antibody levels against $A$. actinomycetemcomitans there were no significant difference between those with coronary disease and those without it ( $4.86 \pm 2.80$ against $4.72 \pm 3.03 \mathrm{EU})$. However, the levels of antibodies to $P$. gingivalis tended to increase in subjects with coronary disease against which did not had it $(7.05 \pm 3.61$ against $6.42 \pm 3.60$ $\mathrm{EU})$.

Therefore coronary diseases were significantly more prevalent among subjects toothed than among toothless population (19.8\% vs. $12.1 \%)$, because the number of teeth was strongly associated with coronary heart disease and antibody levels.

Toothed subjects show a significant difference in higher antibody levels than edentulous subjects.

In the largest studies in which a greater number of scoring variables were considered such as age, sanitary education, weight, number of filled teeth, smoker or not, alcohol consumer, cholesterol, etc., values regarding the prevalence of coronary heart disease were no longer significant.

Therefore they concluded that in a linear regression model there is a direct correlation between periodontal antibody levels and the prevalence of coronary disease. In addition to age coronary disease was just established as a risk factor when the subjects had high cholesterol levels.

Among edentulous subjects the number of remaining teeth was important in terms of antibody values.

They also found that Ig-G antibodies to periodontal pathogens remained elevated after periodontal treatment but decrease when the teeth are extracted. The fact that $13.8 \%$ of edentulous subjects had high levels of antibodies seems to be related to the fact that these patients had recently lost teeth.

The results of the study indicate that the dentition and the presence of high levels of antibodies to $P$. gingivalis respond better against periodontal pathogens associated with the prevalence of coronary heart disease. The present studies suggest that periodontal infection and the host response to infection may play an important role in the pathogenesis of coronary heart disease [22].

The same authors have also studied in 2004 antibody levels of $P$. gingivalis prior to myocardial infarction and thus thought about the possibility of finding an epidemi- 
ological link between the presence of coronary heart disease and periodontitis clinically diagnosed cases.

In the results, it was concluded that there is a significant association between levels of Ig-G or Ig-A and antibody levels against $A$. actinomycetemcomitans and $P$. gingivalis. However, high levels of antibodies against $P$. gingivalis and Ig-A seem to predispose to myocardial infarction regardless of traditional cardiovascular risk factors.

The conclusion was that there was no relationship found between levels of antibodies to A. actinomycetemcomitans and the prevalence to suffer a heart attack, but there is however an increased prevalence with rising levels of antibodies against $P$. gingivalis [23].

It has also been described by Meurmann, et al. (2004) that periodontitis increases by $25 \%$ the risk of cardiovascular disease. And in that sense, they analysed data from more than fifteen independent studies which indicate that periodontitis is a proved risk factor which can contribute to the development of cardiovascular disease. Even some people consider this oral disease may raise the possibility up to $25 \%$. Some bacteria such as $P$. gingivalis, which remain chronically, let this oral infection contribute to the onset of diseases such as cardiovascular disease, respiratory, diabetes and low weight new-borns.

Periodontal disease is a risk factor with such a specific importance as the traditional ones: HTA, cholesterol and obesity, existing a relationship between cytokines and proinflammatory mediators that cause endothelial damage and facilitate the detachment of atherosclerotic plaques.

Sinisalo, J. et al. (2000) published a study of periodontal disease and its association with endothelial dysfunction of the brachial artery and systemic inflammation. These aimed to determine if periodontal disease is associated with endothelial dysfunction and systemic inflammation. They observed 26 subjects with advanced periodontal disease and 29 control subjects. It was observed the dilation of the brachial artery endothelium, mediated dilation by nitro-glycerine and levels of Creactive protein were also examined.

As a result, it was found that patients presenting an advanced periodontal disease had a lower dilatation of the brachial artery endothelium and greater levels of Creactive protein.

Mediated dilation by nitro-glycerine was similar in both groups [24].

Amar et al. (2003) found that endothelial dysfunction is associated with coronary artery disease and coronary risk factors. This study shows that patients with severe periodontal disease show endothelial dysfunction of the brachial artery and high levels of C-reactive protein, which supports previous evidence suggesting a relationship between periodontitis and cardiovascular disease [25].
Tonetti, et al. (2007) shows that deep periodontal treatment in patients with severe periodontitis disease, improves endothelial function of arteries. All participants had no previous evidence of cardiovascular problems.

The study involved 120 patients who were divided into two groups. The control group underwent only supragingival periodontal treatment, while the test group underwent subgingival periodontal treatment including scaling and subgingival tooth extraction with grave prognosis and subgingival application of antimicrobials.

Records were taken from all participants before the treatment, on the following day, and along over the six-month investigation. The records were related to the endothelial function (measurement of brachial artery diameter and blood flow), and biomarker associated with inflammation, endothelial adhesion and coagulation.

The results of this study show after 24 hours periodontal treatment, the dilatation of the brachial artery was significantly lower in the test group patients, as well as the levels of C-reactive protein, interleukin-6, E-selectin, Von Willebrand factor and neutrophil were higher. This means that subgingival periodontal treatment produces an increase of endothelial dysfunction as a consequence of systemic inflammation. However, the parameters taken over the six months of the study showed an improvement in endothelial function and this is a key factor in the prevention of atherosclerosis. Also plasma levels of E-selectin were lower, and any of the group suffered strokes [26].

\section{DISCUSSION}

Ischemic heart disease, consisting of a decrease in myocardial perfusion, is mainly due to arteriosclerosis. Factors that favour its occurrence are age, male sex, hypercholesterolemia, tobacco, diabetes, obesity and chronic infections. Many of these are common risk factors in periodontal disease [27].

Periodontal inflammation can cause a systemic inflammatory response, as evidenced by increases in Creactive protein [28].

Pathogens related to periodontal disease such as $P$. gingivalis, Tannerella forsythensis and A. actiomycetemcomitans have been found in atherosclerotic plaques [29] and there is evidence that $P$. gingivalis can adhere and infect endothelial cells with consequent activation and expression of molecular adhesion cells [30].

These findings make the relationship between periodontal disease and atherosclerosis more evident, since endothelial dysfunction seems to be an early fact in the development of atherosclerosis and it also predicts the instability of the plaque [31] and they show the causal relationship between periodontal disease and atherosclerosis. Moreover, there are other studies reporting that 
individuals with high levels of LDL cholesterol and ischemic heart disease present deeper periodontal pockets than control patients [32].

Beck, et al. (2001) described that in patients with periodontitis and insulin-dependent diabetes may exist an abnormal cytokine secretion, which may present a high risk of ischemic heart disease [33].

Herzberg and Meyer (1996) inoculated rabbits with $S$. sanguis and it was observed a rapid platelet aggregation with alterations in the ECG, blood pressure and the cardiac contractility [34].

Despite all this evidence, there are many authors who have found no relationship between these two pathologies, so Chong, et al. (2000) made a statistical analysis of the studies conducted by Mattila, Joshiopura and Beck, in which they conclude that there is no association between the two pathologies or if there is it is really minor, since the risk factors for both diseases overlap, and therefore, periodontitis may be a phenomenon that occurs parallel to cardiovascular ischemic pathology [35].

On the other hand, studies involving chronic periodontitis as a chronic infection generally do not consider the existence of another infection or chronic inflammation present in patients included in the studies, which could produce a significant bias [36].

Hujoel, et al. (2000) confirmed that there is no evidence to affirm that suffering periodontitis is directly related to the pathology of chronic heart disease. For the amount of people studied (8032 patients) and for the 20 years that lasted the research, this is the most comprehensive and complete study done to date.

The state of their teeth and gums were diagnosed in detail at the baseline of the study.

The researchers also collected other variables as if they were smokers, cholesterol levels and their physical activity.

People who already had some type of heart disease were excluded.

It was concluded that many people suffer from both types of diseases but it is not possible to determine which one causes the other, when both of them have the same risk factors, such as tobacco, stress, socio-economic position, obesity or old age. Periodontitis may coincide with the presence of coronary disease, but it does not necessarily increases the risk of its occurrence. If that was the case, this risk should be reversible, because treating periodontitis would make the probability of inducing coronary disease disappear [37].

Cairo, F. et al. in 2004 conducted a study on periodontal pathogens in atheroma plaques. The objective of this study was to determine the presence or not of periodontal bacterial DNA in carotid atherosclerotic plaques and to calculate the concomitant presence of the same periodontal bacterial DNA, if any, in the periodontal space and carotid atheroma of the same patient.

The study was conducted on 52 patients (26 dentate and 26 edentulous), which were going to undergo surgery because they presented carotid atherosclerotic plaques. Plaque samples and carotid specimens were examined using the technique of the chain reaction of polymerase.

12 of 52 patients were excluded because their negative results for DNA amplification. In the remaining patients periodontal bacteria DNA by PCR was not found in any of the samples.

They concluded that the presence of bacterial DNA, from periodontal origin in atherosclerotic plaques, could not be confirmed in their study and a correlation between species associated with periodontal diseases and bacteria that contribute to the formation of atherosclerotic plaques cannot be established a period [38].

\section{CONCLUSIONS}

Many theories attempt to find a relationship between periodontal disease and ischemic heart disease, and even there are studies in which we can appreciate a clear association between periodontitis, treatment and possible public health implications. However, more studies are needed to determine whether this association actually exists and, if so, it must be determined whether the relationship between periodontitis and coronary heart disease is a cause-effect one and whether the treatment of periodontitis may contribute somewhat to the prevention of atherosclerosis and myocardial infarction.

The association between cardiovascular disease and periodontal infection has been established in several clinical trials, but there may be numerous biases due to risk factors such as age, tobacco, hypercholesterolemia and socio-economic level, among others, that are common to both pathologies and therefore may be a predisposing factor.

\section{REFERENCES}

[1] Lindhe, N.Y. (2000) Exploración de pacientes con enfermedad periodontal. Periodontología clínica e implantología odontológica. Editorial Médica Panamericana, Madrid, 387-398.

[2] Offenbacher, S. (1999) Periodontitis-atherosclerosis syndrome: An expanded model of pathogenesis. Journal of Periodontal Research, 34, 346-352. doi:10.1111/j.1600-0765.1999.tb02264.x

[3] Joshipura, K.J., Rimm, E.B., Douglass, C.W., Trichopoulos, D., Ascherio, A. and Willett, W.C. (1996) Poor oral health and coronary heart disease. Journal of Dental Research, 75, 1631-1636. doi:10.1177/00220345960750090301

[4] Kaski, J.C. (2000) Inflamación, infección y enfermedad 
coronaria: Mitos y realidades. Revista Española de Cardiología, 53, 1311-1317. doi:10.1016/S0300-8932(00)75234-1

[5] Mattila, K.J., Valtonen, V.V., Nieminen, M. and Huttunen, J.K. (1995) Dental infection and the risk of new coronary events: Prospective study of patients with documented coronary artery disease. Clinical Infectious Diseases, 20, 588-592. doi:10.1093/clinids/20.3.588

[6] Wu, T., Trevisan, M., Genco, R.J., Falkner, K.L., Dorn, J.P. and Sempos, C.T. (2000) Examination of the relation between periodontal health status and cardiovascular risk factors: Serum total and high density lipoprotein cholesterol, C-reactive protein, and plasma fibrinogen. American Journal of Epidemiology, 151, 273-282.

doi:10.1093/oxfordjournals.aje.a010203

[7] DeStefano, F., Anda, R.F., Kahn, H.S., Williamson, D.F. and Russell, C.M. (1993) Dental disease and risk of coronary heart disease and mortality. British Medical Journal, 306, 688-691. doi:10.1136/bmj.306.6879.688

[8] Seymour, R.A. and Steele, J.G. (1998) Is there a link between periodontal disease and coronary heart disease? British Dental Journal, 184, 33-38. doi:10.1038/sj.bdj.4809536

[9] Meikle, L.C., Heath, J.K. and Reinolds, J.J. (1986) Advances in understanding cell interactions in tissue resorption. Relevance to the pathogenesis of periodontal diseases and a new hypothesis. Journal of Oral Pathology \& Medicine, 15, 239-250. doi:10.1111/j.1600-0714.1986.tb00616.x

[10] Herzberg, M.C. and Weyer, M.W. (1998) Dental plaque, platelets and cardiovascular diseases. Journal of Periodontology Online, 3, 151-160. doi:10.1902/annals.1998.3.1.151

[11] Genco, D.K. (1998) Invasión of the bucal pathogen porphyromonas gingivalis: Implications for cardiovascular disease. Invasion Metastasis, 18, 57-69.

[12] Meurman, J.H., Sanz, M. and Janket, S.J. (2004) Oral health, atherosclerosis and cardiovascular disease. Critical Reviews in Oral Biology \& Medicine, 15, 403-413. doi:10.1177/154411130401500606

[13] Pussinen, P.J., Jauhiainen, M., Vilkuna-Rautiainen, T., Sundvall, J., Vesanen, M., Mattila, K., Palosuo, T., Alfthan, G. and Asikainen, S. (2004) Periodontitis decreases the antiatherogenic potency of high density lipoprotein. The Journal of Lipid Research, 45, 139-147. doi:10.1194/jlr.M300250-JLR200

[14] Li, L., Messas, E., Batista, E.L. Jr., Levine, R.A. and Amar, S. (2002) Porphyromonas gingivalis infection accelerates the progression of atherosclerosis in a heterozygous apolipoprotein E-deficient murine model. Circulation, 105, 861-867. doi:10.1161/hc0702.104178

[15] Li, J., Helmerhorst, E.J., Leone, C.W., Troxler, R.F., Yaskell, T., Haffajee, A.D., Socransky, S.S. and Oppenheim, F.G. (2004) Identification of early microbial colonizers in human dental biofilm. Journal of Applied Microbiology, 97, 1311-1318. doi:10.1111/j.1365-2672.2004.02420.x
[16] Furuichi, Y., Shimotsu, A., Ito, H., Namariyama, Y., Yotsumoto, Y., Hino, Y., Mishige, Y., Inoue, M. and Izumi, Y. (2003) Associations of periodontal status with general health conditions and serum antibody titers for Porphyromonas gingivalis and Actinobacillus actinomycetemcomitans. Journal of Periodontology Online, 74, 1491-1497. doi:10.1902/jop.2003.74.10.1491

[17] Beck, J., García, R., Heiss, G., Vokonas, P.S. and Offenbacher, S. (1996) Periodontal disease and cardiovascular disease. Journal of Periodontology Online, 67, 11231137.

[18] Emingil, G., Buduneli, E., Aliyev, A., Akilli, A. and Atilla, G. (2000) Association between periodontal disease and acute myocardial infarction. Journal of Periodontology Online, 71, 1882-1886. doi:10.1902/jop.2000.71.12.1882

[19] Loesche, W.J., Schork, A., Terpenning, M.S., Chen, Y.M., Dominguez, B.L. and Grossman, Y.N. (1998) Assessing the relationship between dental disease and coronary heart disease in elderly US veterans. The Journal of the American Dental Association, 129, 301-311.

[20] Takata, Y., Ansai, T., Matsumura, K., Awano, S., Hamasaki, T., Sonoki, K., Kusaba, A., Akifusa, S. and Takehara, T. (2001) Relationship between tooth loss and electrocardiographic abnormalities in octogenarian. Journal of Dental Research, 80, 1648-1652. doi:10.1177/00220345010800071001

[21] Hung, H.C., Willett, W., Merchant, A., Rosner, B.A., Ascherio, A. and Joshipura, K.J. (2003) Oral health and peripheral arterial disease. Circulation, 107, 1152-1157. doi:10.1161/01.CIR.0000051456.68470.C8

[22] Joshipura, K.J., Hung, H.C., Rimm, E.B., Willett, W.C. and Ascherio, A. (2003) Periodontal disease, tooth loss, and incidence of ischemic stroke. Stroke, 34, 47-52. doi:10.1161/01.STR.0000052974.79428.0C

[23] Pussinen, P.J., Jousilahti, P., Alfthan, G., Palosuo, T., Asikainen, S. and Salomaa, V. (2003) Antibodies to periodontal pathogens are associated with coronary heart disease. Arteriosclerosis, Thrombosis, and Vascular Biology, 23, 1250-1264. doi:10.1161/01.ATV.0000072969.71452.87

[24] Sinisalo, J., Paronen, J., Mattila, K.J., Syrjala, M., Alfthan, G., Palosuo, T., Nieminen, M.S. and Vaarala, O. (2000) Relation of inflammation to vascular function in patients with coronary heart disease. Atherosclerosis, 149, 403-411. doi:10.1016/S0021-9150(99)00333-0

[25] Amar, S., Gokce, N., Morgan, S., Loukideli, M., Van Dyke, T.E. and Vita, J.A. (2003) Periodontal disease is associated with brachial artery endothelial dysfunction and systemic inflammation. Arteriosclerosis, Thrombosis, and Vascular Biology, 23, 1245-1249. doi:10.1161/01.ATV.0000078603.90302.4A

[26] Tonetti, M.S., D’Aiuto, F., Nibali, L., Donald, A., Storry, C., Parkar, M., Suvan, J., Hingorani, A.D., Vallance, P. and Deanfield, J. (2007) Treatment of periodontitis and endothelial function. The New England Journal of Medicine, 356, 911-920. doi:10.1056/NEJMoa063186 
[27] Haynes, W.G. and Stanford, C. (2003) Periodontal disease and atherosclerosis: From dental to arterial plaque. Arteriosclerosis, Thrombosis, and Vascular Biology, 23, 1309-1311. doi:10.1161/01.ATV.0000087144.24654.71

[28] Slade, G.D., Offenbacher, S., Beck, J.D., Heiss, G. and Pankow, J.S. (2000) Acute-phase inflammatory response to periodontal disease in the US population. Journal of Dental Research, 79, 49-57. doi:10.1177/00220345000790010701

[29] Haraszthy, V.I., Zambon, J.J., Trevisan, M., Zeid, M. and Genco, R.J. (2000) Identification of periodontal pathogens in atheromatous plaques. Journal of Periodontology Online, 71, 1554-1560.

doi:10.1902/jop.2000.71.10.1554

[30] Deshpande, R.G., Khan, M.B. and Genco, C.A. (1998) Invasion of aortic and heart endothelial cells by Porphyromonas gingivalis. Infection and Immunity, 66, 53375343.

[31] Suwaidi, J.A., Hamasaki, S., Higano, S.T., Nishimura, R.A., Holmes, D.R. Jr. and Lerman, A. (2000) Long-term follow-up of patients with mild coronary artery disease and endothelial dysfunction. Circulation, 101, 948-954. doi:10.1161/01.CIR.101.9.948

[32] Katz, J., Flugelman, M.Y., Goldberg, A. and Heft, M. (2002) Association between periodontal pockets and elevated cholesterol and low density lipoprotein cholesterol levels. Journal of Periodontology Online, 73, 494-500. doi:10.1902/jop.2002.73.5.494
[33] Beck, J.D. and Offenbacher, S. (2001) The association between periodontal diseases and cardiovascular diseases: A state-of-the-science review. Journal of Periodontology Online-Annals of Periodontology, 6, 9-15. doi:10.1902/annals.2001.6.1.9

[34] Herzberg, M.C. and Meyer, M.W. (1996) Effects of oral flora on platelets: Possible consequences in cardiovascular disease. Journal of Periodontology Online, 67, 11381142. doi:10.1902/jop.1996.67.10s.1138

[35] Chong, P.H. and Kezele, B. (2000) Periodontal disease and atherosclerotic cardiovascular disease: Confounding effects or epiphenomenon? Pharmacotherapy, 20, 805818. doi:10.1592/phco.20.9.805.35189

[36] Armitage, G.C. (2000) Periodontal infections and cardiovascular disease-how strong is the association? Oral Diseases, 6, 335-350. doi:10.1111/j.1601-0825.2000.tb00126.x

[37] Hujoel, P.P., Drangsholt, M., Spiekerman, C. and De Rouen, T.A. (2000) Periodontal disease and coronary heart disease risk. Journal of the American Medical Association, 284, 1406-1410. doi:10.1001/jama.284.11.1406

[38] Cairo, F., Gaeta, C., Dorigo, W., Oggioni, M.R., Pratesi, C., Pini Prato, G.P. and Pozzi, G. (2004) Periodontal pathogens in atheromatous plaques. A controlled clinical and laboratory trial. Journal of Periodontal Research, 39, 442-446. doi:10.1111/j.1600-0765.2004.00761.x 